\title{
Appropriate sampling strategies to estimate sea lice prevalence on salmon farms with low infestation levels
}

Jaewoon Jeong a, *, Crawford W. Revie a, b

${ }^{a}$ Department of Health Management, Atlantic Veterinary College, University of Prince Edward Island, Charlottetown, 550 University Ave, Charlottetown, PE, C1A 4P3, Canada

${ }^{\mathrm{b}}$ Department of Computer and Information Sciences, University of Strathclyde, Glasgow, G1 1XQ, UK

* Corresponding author. Tel.: +1 902566 0579; Fax: +1 9026205053

E-mail address: jjeong@upei.ca (Jaewoon Jeong)

Running title: Sampling salmon to estimate sea lice prevalence 


\begin{abstract}
Effective sampling is essential to monitoring and controlling sea lice infestations on salmon farms. However, official sampling regimes are often inadequate, typically adopting a one-sizefits-all approach. Over the past decade, the thresholds at which mandatory treatment is required has also been reduced in many regions and, as such, the total infestation loads reported from salmon farms tend to be lower. Therefore, the use of prevalence, as opposed to the more conventionally used abundance, becomes a metric of interest and, from an analytical perspective, offers some beneficial characteristics. This paper explores a range of sampling scenarios and their impacts on the accuracy of sea lice estimation, particularly when prevalence is the adopted metric.

Empirical sea lice count data demonstrated a good fit to the negative binomial distribution and provided a probable range of values that could be used to describe typical levels of overdispersion. It was demonstrated that, when prevalence is low, it can be reliably used to predict abundance. Monte Carlo simulations of a hypothetical salmon farm were then used to test results for a variety of sample sizes and sea lice infestation scenarios. Different sea lice infestation levels between pens in a farm (i.e. a spatial clustering effect) and aggregations of sea lice on their hosts (i.e. the effect of over-dispersion) were simulated to explore a variety of conditions. The extent to which higher levels of clustering and/or over-dispersion necessitate the need for larger sample sizes to achieve similar levels of accuracy was explored. The level of accuracy that can be achieved in practice depends on many factors and what is considered to be an acceptable level of accuracy will, by definition, be subjective and vary according to the purpose for which the estimation is being carried out. This study includes a variety of possible situations to guide farm operators in choosing sample sizes according to their particular requirements. Furthermore, appropriate sample size determination can be expected to reduce overall sampling effort, achieve better overall control, help avoid unnecessary treatments, and reduce both associated costs and fish welfare impacts.
\end{abstract}

Keywords: clustering; negative binomial distribution; prevalence; salmon aquaculture; sample size; sea lice

Jeong \& Revie, 2020 (Sampling salmon to estimate sea lice prevalence) 


\section{Introduction}

Sea louse (Lepeophtheirus salmonis and Caligus spp.) infestation has resulted in substantial economic losses and is a serious concern for salmon aquaculture (Johnson et al., 2004). Costs associated with sea lice infestation were, for example, estimated to account for $9 \%$ of total farm revenue for a typical spring-release cycle in central Norway (Abolofia et al., 2017). Sea lice spread from salmon farms is also of broader ecological concern, with putative links to wild salmonid declines (Krkosek et al., 2005; Costello, 2009a). In addition, welfare of salmon can be seriously impacted by sea lice infestation (Overton et al., 2018). These economic, conservation and welfare issues are key drivers for an increased interest in controlling sea louse infestation on salmon farms.

The control of sea lice relies on effective monitoring programs based on on-going salmon sampling, which allows farm operators to estimate sea lice infestation levels on their farms (Revie et al., 2005). The accurate estimation of sea lice burdens on salmon farms is required to ensure that treatments are administered appropriately and that any relevant regulatory thresholds are being adhered to. In addition, subsequent to a treatment, the success or otherwise of that intervention can only be confirmed if the sampling protocol is adequate (Gautam et al., 2017). Therefore, reliable surveillance is a prerequisite to better controlling sea lice populations, and ultimately to creating more sustainable salmon aquaculture (Groner et al., 2016). However, guidelines as to the appropriate sampling approaches needed to achieve accurate sea lice load estimates remain largely under-developed, in part due to the lack of comprehensive data on sea lice infestation distributions on farmed salmon under varying real-world settings.

Critical to determining adequate sample size is an understanding of the potential impacts of host population clustering on parasite distribution (McDermott and Schukken, 1994). A given salmon farm consists of multiple pens, and what salmon farm operators often wish to assess through sampling is the overall sea lice load at the farm level, rather than at the pen level. For a given total sample size, sampling from fewer pens is less logistically challenging and consequently requires less effort. As a result, salmon farms have historically estimated sea lice load over the whole site by sampling from a limited number of pens. However, it has been demonstrated that sea lice abundance on salmon farms can vary significantly among pens (Revie et al., 2005; Revie et al., 2007), and Revie et al. (2005) emphasized that sampling a smaller number of fish from a larger number of pens resulted in a more accurate estimate of abundance. Such clustering of sea lice should therefore not be disregarded when determining the sample size needed to estimate sea lice infestation. Indeed, sampling protocols in a number of regions now reflect this understanding, requiring that the majority of pens within a site be regularly sampled (Norwegian Ministry of Industry and Fisheries, 2017).

Three metrics can be used to quantify sea lice infestation levels: prevalence, abundance, and intensity. Abundance is defined as the average number of sea lice per fish, prevalence as the 
proportion of fish with one or more sea lice, and intensity as the average number of sea lice per infested fish (Bush et al., 1997). Of these three metrics, abundance has been the most widely used in aquaculture (Baillie et al., 2009), and the levels at which sea lice treatment is triggered in various salmon-producing countries tend to depend on this measure of infestation (Johnson et al., 2004). However, it has been argued that abundance can be fairly accurately predicted given a measurement of prevalence (Baillie et al., 2009; Heuch et al., 2011). In the cases of sea lice counts on Scottish salmon farms (Baillie et al., 2009) and wild pink juvenile salmon in BC, Canada (Jones and Hargreaves, 2009), a linear relationship was shown to exist between abundance and prevalence when prevalence was relatively low (approximately $\leq 50 \%$ ) (Heuch et al., 2011). A similar result was demonstrated for a much larger number of wild juvenile samples in BC, Canada (Patanasatienkul et al., 2013), where a linear relationship was seen to hold for values of up to approximately $60 \%$ prevalence.

Baillie et al. (2009) emphasized that sampling effort could be significantly reduced by recording the presence or absence of sea lice (prevalence), rather than counting the number of parasites (abundance). They also noted that newer forms of observation technology, such as image analyses, may enable presence or absence to be detected more effectively than would be possible if the aim was to determine actual sea lice numbers (Baillie et al., 2009). From a fish welfare perspective, estimating prevalence rather than abundance is also expected to reduce the amount of time that fish are required to be out of the water. It is therefore important to better understand the relationship between sea lice abundance and prevalence on salmon to assess the potential, as well as any limitations, of using prevalence as the primary infestation metric.

If sea lice randomly choose their host, the statistical distribution of parasite counts per host should follow a Poisson distribution, where the variance would be approximately equal to the mean. However, it has often been observed that parasites aggregate on some portion of available hosts, leading to an over-dispersed parasite count distribution (i.e. where variance is significantly greater than the mean) (Anderson and May, 1992). Over-dispersion in ecological count data can be modelled well using the negative binomial distribution (Lindén and Mäntyniemi, 2011), and has been applied elsewhere in the context of sea lice on salmon hosts (Treasurer, 2000; Penston et al., 2008; Aldrin et al., 2013). The negative binomial distribution can be applied to sea lice abundance such that $\mathrm{NB}(\kappa, \mathrm{A})$, is a function of $\mathrm{A}$, the number of sea lice per salmon (mean abundance), and $\kappa$, a parameter to represent the level of dispersion, which explains the shape of the statistical distribution. Since fish will either be infested with sea lice or not, sea lice prevalence can be described by a binomial distribution, $B(n, p)$, where $n$ is the total number of salmon and $p$ is the probability of infestation with at least one sea louse; $n^{*} p$ then becomes prevalence. Estimating prevalence based on knowledge of the modelled abundance is straightforward, whereas estimating abundance based on prevalence is more complicated due to the lack of any explicit definition of $\kappa$ in the binomial distribution. Depending on $\kappa$, a variety of abundance distributions are possible. It is therefore critical to consider the range of abundances that might be estimated based on a given level of prevalence under a variety of realistic assumptions as to the value of $\kappa$ (Lloyd-Smith, 2007). 
This study consists of two parts: i) an analysis of empirical data, and ii) a Monte Carlo simulation. First, we use empirical data to confirm that prevalence, in contrast to the more typically adopted metric of abundance, can be used as an effective method for measuring levels of sea lice infestation in aquaculture. This confirmation should depend on the finding of a universal value of dispersion parameter of negative binomial distribution of sea lice abundance in farmed salmon. Second, we adopt the Monte Carlo method to simulate salmon sampling events on a hypothetical salmon farm for two purposes. One purpose is to explore how different sampling strategies affect the accuracy of sea lice prevalence estimates. The importance of accurate sea lice infestation estimation is highlighted by the fact that, in many production contexts, intervention is required once sea lice reach a certain level (Johnson et al., 2004). Another purpose is to determine how measures of sea lice treatment effectiveness might vary as a function of estimated differences in sea lice prevalence before and after treatment. Advice regarding appropriate sample size to determine treatment effectiveness can contribute to enhancing the efficiency of treatment and may also help prevent unnecessary treatment application (Gautam et al., 2017).

\section{Materials and Methods}

\subsection{Empirical data analyses}

Sea louse count datasets were collected from Atlantic salmon (Salmo salar) farms of two areas in Norway (Hitra and Hardangerfjord), one area in New Brunswick (NB), eastern Canada, and one area in Chile. Sea lice in Norway and Canada were L. salmonis, while sea lice counted on salmon in Chile were $C$. rogercresseyi. In Norway, sea lice counts were analysed for each pen of each farm, whereas in NB and Chile, sea lice counts from entire farms were pooled for analysis, as only ten fish were sampled from each pen in most cases. Specifically, the data from around Hitra Island, Norway included sea lice counts for 40 fish from each of four pens at one site and from each of five pens at a second site. The data from Hardangerfjord, Norway included sea lice counts for 80 fish from each of nine pens at a site. The data from eastern Canada included sea lice counts for 40 to 120 fish from 12 different sites, while the Chilean data included sea lice counts for 352 fish from seven sites. Samples within each area were collected at the same times, except for in Hitra, Norway, where fish were sampled on three separate occasions over a single week.

We assessed whether the sea lice counts in the datasets obtained from the four areas were consistent with the assumptions of the negative binomial distribution in two ways. First, we estimated an Akaike information criterion (AIC) model fit to the negative binomial and Poisson distributions by using the fitdist function from the fitdistrplus package of R (Delignette-Muller and Dutang, 2015), which is based on maximum likelihood estimation. Second, we performed chi-square tests based on the number of sea lice per fish at the four sites, to assess whether either 
the Poisson or negative binomial distribution best fit the data.

After the assessment, the dispersion parameter $(\kappa)$ of the negative binomial distribution was estimated using a non-linear least squares approach ( $n l s$ function in $\mathrm{R}$ ), which approximates the non-linear equation by minimizing the squared residual error. To fit non-linear functions to the empirical sea lice abundance and prevalence data, we used Equation 1 (Anderson and May, 1985).

$$
\text { prevalence }=1-\left(1+\frac{\text { abundance }}{\kappa}\right)^{-\kappa}
$$

\subsection{Simulation model description}

We set up a hypothetical salmon farm consisting of ten pens, where each pen contained 10,000 salmon, and used the Monte-Carlo method to generate 10,000 iterations of each scenario. In order to simulate different scenarios we had to determine the prevalence of each pen in a farm. The prevalence of each pen can be determined by two positive shape parameters ( $\alpha$ and $\beta)$ of the beta distribution. The beta distribution is a continuous probability distribution between zero and one, parametrized by these shape parameters, and was used to model the set of prevalence values across the pens in a farm. The mean $(\mu)$ and standard deviation $(\sigma)$ of prevalence values for all pens in a farm was used to calculate $\alpha$ and $\beta$ based on the following two equations (Fosgate, 2007):

$$
\begin{gathered}
\alpha=\left(\frac{1-\mu}{\sigma^{2}}-\frac{1}{\mu}\right) \mu^{2} \\
\beta=\alpha\left(\frac{1}{\mu}-1\right)
\end{gathered}
$$

We used intraclass correlation (ICC) to explore different levels of variance in prevalence among pens on a farm. A high ICC value indicates a high level of variance in prevalence among pens in a farm, whereas low ICC indicates low variance in prevalence among pens. For given values of

ICC and $\mu$, we could then determine $\sigma$ such that $\alpha$ and $\beta$ resulted in a range of ICC values, based on the relationship captured in Equation 4 (Fosgate, 2007).

$$
\text { ICC }=\frac{1}{\alpha+\beta+1}
$$

The estimated values of $\mu$ and $\sigma$ were used to obtain $\alpha$ and $\beta$, which we then used with the beta distribution to stochastically determine the prevalence at each pen in a farm. The binomial distribution is a discrete probability distribution, characterised by only two possible outcomes (here, infestation or non-infestation), and was used to model the prevalence within each pen. After the prevalence had been stochastically determined for each pen in each iteration, abundance was determined using Equation 1. 
We set up sampling simulations to explore scenarios that addressed the two main purposes of this study: (i) to estimate the accuracy of sea lice prevalence estimation; and (ii) to evaluate the effects of treatment. For the first goal, accuracy was expressed as the mean deviation of prevalence measured through sampling compared to the true prevalence over many iterations. The deviations were calculated for scenarios comprising between one and ten pens each with between one and 40 fish sampled per pen (i.e. a total of 400 scenarios). The deviations were

calculated as $\frac{\mid \text { (Estimated value through sampling)-(True value }) \mid}{\text { True value }}$. The deviation was expressed as accuracy, in that this deviation represents how close the sampling results are to the true value (Dohoo et al., 2003). This is not to be confused with precision, or how consistent the sampling results might have been.

For the second goal, simulation was used to determine the minimum number of sampled fish required to support the detection of a given difference in sea lice prevalence before and after a treatment. A treatment was considered to be significantly effective in reducing sea lice prevalence if $80 \%$ of the iterations showed a lower prevalence after the treatment than before the treatment (i.e., we used a statistical power of 0.8 ). Three levels of treatment efficacy were used in the simulations, $90 \%, 60 \%$ and $30 \%$.

The efficacy was assumed to be $\left(1-\frac{\text { post-treatment prevalence }}{\text { pre-treatment prevalence }}\right) \times 100 \%$ (Lees et al., 2008). For example, $90 \%$ treatment efficacy means that for 10 salmon that have one or more sea lice prior to treatment, 9 will have no sea lice after treatment.

Irrespective of the specific study goals, the simulations explored a range of prevalence and ICC values. We assumed three levels of true prevalence for a whole farm $(0.1,0.3$, and 0.5$)$, choosing levels from the lower end of the full possible range of prevalence. Because sampling represents proportions in one of two states - infested or not infested - choosing values over 0.5 would generate symmetrical results. Three ICC levels $(0.04,0.16$, and 0.36$)$ were chosen from the range of empirical values reported in Scottish and Norwegian data (Revie et al., 2007). We used all probable combinations of true prevalence and ICC levels, because no significant relationship was previously found to exist between sea lice infestation level and ICC value (Revie et al., 2007). Models in this study did not consider biological characteristics of the sea lice or salmon, such as sex, developmental stage, or weight. Modelling was based on Monte-Carlo simulation using the R platform (R Core Team, 2017).

\section{Results}

\subsection{Empirical data analyses}

In terms of the most appropriate distribution to use for the data considered here, the negative binomial and Poisson distributions did not differ significantly in many cases. The Poisson distribution, for which variance is equal to mean, is a particular case of negative binomial distribution. Thus, if the mean and variance of sea lice counts are significantly similar to each 
other, it is more straightforward to use the Poisson distribution, however if the variance is significantly greater than the mean, the negative binomial distribution will more accurately describe the counts. As can be seen in Fig. 1, for sampling points across four locations, the $\triangle \mathrm{AIC}$ $\left(\mathrm{AIC}_{\mathrm{nb}}-\mathrm{AIC} \mathrm{C}_{\mathrm{P}}\right)$ value was close to zero. However, there were quite a few cases where the $\triangle \mathrm{AIC}$ value was around -100 , and two cases with much larger negative values. These points all occur where the value of $\kappa$ is low, indicating a high level of over-dispersion. When $\kappa$ is high (limited over-dispersion, with approximately equal mean and variance values) the fit provided by the Poisson distribution is almost as good as that obtained by assuming a negative binomial distribution, reflecting the theoretical relationship that would be expected between overdispersion and fit to the negative binomial distribution (McCullagh and Nelder, 1989). In addition, we performed a chi-squared test to see whether $\mathrm{AIC}_{\mathrm{nb}}$ and $\mathrm{AIC}_{\mathrm{P}}$ were significantly different.

In the two Norwegian sites, at which the mean abundances were 0.54 and 0.62 , the P-values were 1 and 0.99, while in NB, Canada and Chile, where the mean abundances were 2.36 and 2.75, the p-values were 0.27 and $<0.001$, respectively. This finding implies that low abundance values in the two Norwegian sites fit both to the Poisson and negative binomial distributions, but that high abundance values such as those found in the sites in NB, Canada and Chile can be fit only to the negative binomial distribution. In addition, the chi-square test generated $p$-values of $<0.001$ and 0.046 for the two Norwegian sites and $<0.001$ for the sites in both NB, Canada and Chile. This result indicates that it is statistically reasonable to regard that the number of sea lice per farmed salmon as following a negative binomial distribution, rather than a Poisson distribution.

We used non-linear least squares to estimate the value of $\kappa$ from the farms in the four locations. The observed relationship between abundance and prevalence for the sea lice samples taken from these four locations followed a pattern similar to the theoretical prediction (i.e. Equation 1) and the best fit was found when the $\kappa$ value was 2.19 (Fig. 2). This fit indicates that the relationship between these two metrics is essentially linear up to abundance levels of around two sea lice (and $\sim 70 \%$ prevalence), but strongly curvilinear thereafter (with an obvious asymptote at $100 \%$ prevalence).

\subsection{Simulation}

\subsubsection{Modelling the effect of various sampling scenarios on sea lice infestation estimates}

Simulated results from a range of scenarios involving different combinations of fish sampled per pen and number of pens sampled were explored in terms of the proportional deviation in the estimated prevalence based on a given sampling strategy from the true prevalence. Not surprisingly, at a prevalence of 0.5 it was possible to achieve estimates that deviated less from the true prevalence than was the case as the prevalence fell to 0.1 (Fig. 3). For example, in the top right-hand panel of Fig. 3 , the contour line labelled " 0.1 " indicates that the absolute 
difference in value between estimated prevalence and true prevalence was 0.05 and the true prevalence was 0.5 (i.e. $0.05 / 0.5=0.1$ ). Looking at a roughly similar location of a contour line in the top-left panel, indicates that a proportional deviation of around $30 \%$ (i.e. $0.03 / 0.1=0.3$ ) would be expected to occur with the same combination of fish sample size per pen and number of pens sampled. The black dotted lines in Fig. 3 indicates the contours of equal total sample size (i.e. ranging from 4 fish from 10 pens, to 40 fish from a single pen). By comparing this curve to the various contours, the known benefit of sampling fewer fish from more pens can be seen, with this effect becoming more obvious as the level of clustering among pens increases (i.e. the higher the modelled ICC values, moving top to bottom in the panels of Fig. 3). In the case of the highest ICC value (0.36), the deviation contours are predominantly affected by the number of pens sampled. It is also clear that, as the ICC increases, larger sample sizes are required to limit the deviation between estimated and true prevalence. In the most challenging scenario explored (bottom-left panel of Fig. 3, with a true prevalence of 0.1 and an ICC $=0.36$ ), even the most extensive sampling strategies would result in estimates with approximately $50 \%$ deviation from the true value (the " 0.5 " contour); a level that would equally be achieved by taking 7 fish from each of the 10 pens, or around 25 fish from each of 9 pens (i.e. at this level of clustering, selecting more fish per pen adds almost no value in terms of improving the prevalence estimate, as the uncertainly can only be resolved by comparing values across the dissimilar pens).

\subsubsection{Modelling the effect of sampling choices on detecting differences in sea lice infestation levels following treatment}

Minimum sample sizes needed to detect a given difference in sea lice prevalence before and after a treatment were explored assuming different levels of true prevalence before a treatment and varying ICC. As the true pre-treatment prevalence approached a value of 0.1 , larger sample sizes were required to detect differences after the treatment (Fig. 4). With a low ICC, the combinations of individual fish sampled per pen and the number of pens sampled was roughly congruent to the curve representing a total of 40 sampled fish. Conversely, when there was a high degree of clustering $(\mathrm{ICC}=0.36$ ), the number of pens needed to be sampled tended to be almost constant regardless of the number of fish sampled per pen, except when the number of fish sampled per pen was markedly low (i.e., $<4)$.

\section{Discussion}

The proposition that, under certain conditions, prevalence may be as good, or better, a metric to use when monitoring sea lice infestation levels on salmon farms (Baillie et al., 2009; Heuch et al., 2011) has needed more examinations of the relation between prevalence and abundance. As such the study explored, by means of empirical data and simulated experiments, the validity of this proposition and best practices that can inform prevalence estimation strategies, depending on the purpose and desired accuracy of sampling. To this end, we first explored the empirical data of sea lice counts per fish on various sites to confirm that the counts follow negative binomial 
distribution. Then, we assessed the dispersion parameter (mean of 2.19) of negative binomial distribution and found that various levels of abundance across sites can be described by a reasonably narrow range of dispersion parameter. This finding allows us to predict abundance through prevalence. Based on this finding, we used prevalence as a metric of sea lice infestation level for Monte-Carlo simulations. Varying values for ICC and prevalence were used to estimate the sample sizes necessary to assess sea lice infestation levels and to detect differences in infestation levels following a course of treatment.

Statistical theory suggests that when prevalence is high a wide range of abundance levels may be present, in contrast to the fairly narrow range of abundance values that will characterise any situation described by low prevalence. The empirical data presented here confirmed this to be the case, indicating a clear and linear relationship up to an abundance of around 2 sea lice (and a prevalence of $\sim 0.7$ ). As a result, it is entirely possible to use prevalence as a substitute for abundance in situations where the overall infestation level on a farm is low. This finding aligns well with previously published results (Heuch et al., 2011; Samsing et al., 2014), further suggesting the generalizability of these observations. Thus, in jurisdictions where low treatment trigger thresholds are in place (e.g. the values of $0.2 / 0.5$ for adult females in Norway; (Norwegian Ministry of Industry and Fisheries, 2017)), there is no apparent reason that prevalence could not be used just as reliably as abundance. Although treatment trigger levels are currently based on abundance (Revie et al., 2009), a shift towards using prevalence might be valuable. Prevalence may indicate pathogenicity at the population level, while abundance may be better suited to measuring pathogenicity at the level of the individual (Costello, 2006). Reporting sea lice infestation levels in terms of both abundance and prevalence may be desirable for a more complete representation of sea lice infestation characteristics on salmon farms. Given the clear linear relationship that appears to hold in many modern salmon farming settings, measuring prevalence alone should suffice to allow estimates of both metrics to be reported, and could be expected to result in savings both in terms of sampling effort and cost (Baillie et al., 2009).

Since the likely variation in sea lice number among salmon pens was first suggested by Treasurer et al. (2000), the evidence of such clustering on Scottish and Norwegian salmon farms has been reported from empirical studies (Revie et al., 2005; Revie et al., 2007), with very similar sea lice clustering characteristics also being observed on farms in Canada (Heuch et al., 2011). Based on these findings, it was demonstrated that sampling "few fish from many pens" was typically a better strategy than sampling "many fish from few pens" (Revie et al., 2007). In this study, we have further illustrated the impact of choosing numbers of fish and of pens to sample under varying levels of infestation and differing degrees of dispersion $\left(\sigma^{2} / \mu\right)$. While these results conform to earlier theoretical hypotheses, the simulated results provide a more detailed understanding of the implications of different sampling strategies, particularly in the context of estimating prevalence levels. While recent studies in Norway have demonstrated that pen level variation can still be significant (Marques et al., 2018), over the past 2-3 years in Norway it has become more common to count sea lice in all pens on a farm (Norwegian Ministry of Industry and Fisheries, 2017) and indeed to use the pen as the unit of assessment for treatment (Groner et 
al., 2019). In this setting the issue of ICC becomes something of a moot point. However, in many other jurisdictions this is not the case (for example, in Chile only four pens are required to be monitored (SERNAPESCA, 2015) and as such pen-level clustering must be factored into any assessments around the accuracy of sea lice infestation estimates.

The reality of parasite aggregation on hosts has been discussed in many previous studies (Anderson and May, 1978; Shaw and Dobson, 1995; Poulin, 2007; Gourbière et al., 2015), and the case of sea lice on salmon is no different from this well-accepted phenomenon (Murray, 2002; Costello, 2006). This study demonstrates the considerable effect of such aggregation on necessary sample size, and emphasizes the need to consider the degree of over-dispersion of sea lice on salmon when assessing the accuracy of prevalence or abundance estimates. The fact that $\kappa$ over-estimation is much more likely than $\kappa$ under-estimation (Lloyd-Smith, 2007) needs to be kept in mind, meaning that the actual levels of sea lice aggregation on salmon is likely higher than has been estimated from empirical data. Thus, the validation of a relatively well-defined range of $\kappa$ values is pre-requisite when estimating prevalence from abundance (particularly high abundance) or vice versa. Another important caution is our assumption that salmon are randomly sampled from the population in their respective pens. However, this assumption can seldom be guaranteed or even expected in real situations. Randomly sampling farmed salmon for something as relatively simple as estimating their size has been demonstrated to be difficult to achieve (Nilsson and Folkedal, 2018). Therefore, sample sizes found here should probably be adjusted upwards to achieve a desired level of accuracy in sea lice level estimation. We also need to better understand the relationship between sea lice infestation levels and the probability of an infested host being sampled, in order to properly estimate the true degree of sea lice aggregation on salmon. However, in situations of low average prevalence (typically with many fewer than 2 adult sea lice per host) it is highly unlikely that farmed salmon will suffer any physiological effects that might be evidenced in swimming or other behaviours which could increase their likelihood of being sampled.

Farms are required to report mean sea lice counts per fish (abundance) in most salmon producing regions, with further sampling being carried out by public officials in some regions (Revie et al., 2009; Elmoslemany et al., 2013). Sampling outcomes that indicate sea lice abundance close to or above treatment trigger levels will result in significant treatment costs. Thus, exact and reliable estimates of sea lice infestation levels through appropriate salmon sampling are crucial. Also, even where effective controls are available, treatment cost is still ranked as being the sea licerelated effect to have the greatest impact on the profitability of salmon farms (Costello, 2009b). Treatment costs can be saved by intervening only when necessary, and the true effectiveness of any treatments applied can be better measured if appropriate sampling strategies are put in place. The prevention of unnecessary treatment, caused by inappropriate sampling strategies, would also contribute to sea lice control in the longer term by delaying the development of sea lice resistance to parasiticides (Costello, 2006; Aaen et al., 2015). Chemical treatments that may impact non-target crustaceans would also be reduced (Waddy et al., 2002). In addition, fish welfare can be improved by ensured that only necessary treatments are performed. Concerns 
have been raised regarding fish welfare for a number of the more recently deployed nonmedicinal treatments (Overton et al., 2018).

Despite the importance of sampling, it is not trivial to devise the most effective strategies. As this study has illustrated, understanding infestation levels, the degree of over-dispersion and clustering effects among pens on farms are all critical to successful sampling. It is thus perhaps unsurprising that regulators have not engaged in more nuanced definition of treatment thresholds or alternative metrics for reporting. Information to support a successful sampling plan, can itself require significant sampling to calibrate the basic distributional characteristics. Therefore, compiling and making available databases from farms under varying conditions can be extremely valuable to farm operators as they seek to better understand and predict the infestation situation on any given farm.

The results of the simulations carried out within this study could have been presented in a number of ways. For sampling accuracy, we reported the deviation of estimated mean sea lice prevalence levels between sampled and true infestation levels. It would also have been reasonable to estimate the likelihood that the estimated prevalence level lay within a certain range of the true infestation level. The reason we presented the simulation results in terms of deviation, is that we believe that a focus on lack of accuracy (deviations) is more likely to motivate an interest in and discussion of the numerous external and internal factors, such as salmon or sea lice species, farm conditions, federal regulations, and farm operators' opinions (Torrissen et al., 2013) that must be accounted for in any attempt to improve sea lice infestation estimation on salmon farms. Should regulatory mechanisms such as the 'Traffic Light' framework recently adopted in Norway (Vollset et al., 2017) become the norm in other jurisdictions, the need to more accurate and dependable sea lice estimation approaches will only increase over time. 


\section{Conflict of interest statement}

No conflict of interest to declare.

None of the authors have a financial or personal relationship with other people or organizations that could inappropriately influence (bias) our work.

\section{Acknowledgements}

This work was partially funded through an FHF Norway research grant (FHF 901411 Developing standardised methods for counting seal lice and calculating the amounts of sea lice) and by the Ocean Frontier Institute's New Models of Salmon Health Management (OFI Module L) programme. We also thank Stephanie Palmer for editorial assistance with this manuscript. 


\section{References}

Aaen, S.M., Helgesen, K.O., Bakke, M.J., Kaur, K., Horsberg, T.E., 2015. Drug resistance in sea lice: a threat to salmonid aquaculture. Trends in Parasitology 31, 72-81.

Abolofia, J., Asche, F., Wilen, J.E., 2017. The cost of lice: quantifying the impacts of parasitic sea lice on farmed salmon. Marine Resource Economics 32, 329-349.

Aldrin, M., Storvik, B., Kristoffersen, A.B., Jansen, P.A., 2013. Space-time modelling of the spread of salmon lice between and within Norwegian marine salmon farms. PLoS One 8, e64039.

Anderson, R.M., May, R.M., 1978. Regulation and stability of host-parasite population interactions: I. Regulatory processes. The Journal of Animal Ecology, 219-247.

Anderson, R.M., May, R.M., 1985. Helminth infections of humans: mathematical models, population dynamics, and control. Advances in Parasitology. Elsevier, 1-101.

Anderson, R.M., May, R.M., 1992. Infectious diseases of humans: dynamics and control. Oxford University Press.

Baillie, M., Lees, F., Gettinby, G., Revie, C., 2009. The use of prevalence as a measure of lice burden: a case study of Lepeophtheirus salmonis on Scottish Atlantic salmon, Salmo salar L., farms. Journal of Fish Diseases 32, 15-25.

Bush, A.O., Lafferty, K.D., Lotz, J.M., Shostak, A.W., 1997. Parasitology meets ecology on its own terms: Margolis et al. revisited. The Journal of Parasitology, 575-583.

Costello, M.J., 2006. Ecology of sea lice parasitic on farmed and wild fish. Trends in Parasitology 22, 475-483.

Costello, M.J., 2009a. How sea lice from salmon farms may cause wild salmonid declines in Europe and North America and be a threat to fishes elsewhere. Proceedings of the Royal Society B: Biological Sciences 276, 3385-3394.

Costello, M.J., 2009b. The global economic cost of sea lice to the salmonid farming industry. Journal of Fish Diseases 32, 115-118.

Delignette-Muller, M.L., Dutang, C., 2015. fitdistrplus: An R package for fitting distributions. Journal of Statistical Software 64, 1-34.

Dohoo, I.R., Martin, W., Stryhn, H., 2003. Veterinary epidemiologic research. AVC Incorporated Charlottetown, Canada.

Elmoslemany, A., Whyte, S.K., Revie, C.W., Hammell, K.L., 2013. Sea lice monitoring on Atlantic salmon farms in New Brunswick, Canada: comparing audit and farm staff counts. Journal of Fish Diseases 36, 241-247.

Fosgate, G., 2007. A cluster-adjusted sample size algorithm for proportions was developed using 
a beta-binomial model. Journal of Clinical Epidemiology 60, 250-255.

Gautam, R., Vanderstichel, R., Boerlage, A., Revie, C., Hammell, K., 2017. Evaluating bath treatment effectiveness in the control of sea lice burdens on Atlantic salmon in New Brunswick, Canada. Journal of Fish Diseases 40, 895-905.

Gourbière, S., Morand, S., Waxman, D., 2015. Fundamental factors determining the nature of parasite aggregation in hosts. PLoS One 10, e0116893.

Groner, M.L., McEwan, G.F., Rees, E.E., Gettinby, G., Revie, C.W., 2016. Quantifying the influence of salinity and temperature on the population dynamics of a marine ectoparasite. Canadian Journal of Fisheries and Aquatic Sciences 73, 1281-1291.

Groner, M.L., Laurin, E., Stormoen, M., Sanchez, J., Revie, C.W., 2019. Potential for sea lice to evolve freshwater tolerance as a consequence of freshwater treatments in salmon aquaculture: a literature review. Aquaculture Environment Interactions (in press)

Heuch, P.A., Gettinby, G., Revie, C.W., 2011. Counting sea lice on Atlantic salmon farmsempirical and theoretical observations. Aquaculture 320, 149-153.

Johnson, S.C., Bravo, S., Nagasawa, K., Kabata, Z., 2004. A review of the impacts of parasitic copepods on marine aquaculture. Zoological Studies 43, 8-19.

Jones, S.R., Hargreaves, N.B., 2009. Infection threshold to estimate Lepeophtheirus salmonisassociated mortality among juvenile pink salmon. Diseases of Aquatic Organisms 84, 131-137. Krkosek, M., Lewis, M.A., Volpe, J.P., 2005. Transmission dynamics of parasitic sea lice from farm to wild salmon. Proceedings of the Royal Society B: Biological Sciences 272, 689-696.

Lees, F., Baillie, M., Gettinby, G., Revie, C.W., 2008. The efficacy of emamectin benzoate against infestations of Lepeophtheirus salmonis on farmed Atlantic salmon (Salmo salar L) in Scotland, 2002-2006. PLoS One 3, e1549.

Lindén, A., Mäntyniemi, S., 2011. Using the negative binomial distribution to model overdispersion in ecological count data. Ecology 92, 1414-1421.

Lloyd-Smith, J.O., 2007. Maximum likelihood estimation of the negative binomial dispersion parameter for highly overdispersed data, with applications to infectious diseases. PLoS One 2, e180.

Marques, A.R., Forde, H., Revie, C.W., 2018. Time-series clustering of cage-level sea lice data. PloS One 13, e0204319.

McCullagh, P., Nelder, J., 1989. Generalized Linear Models (Second ed.). Boca Raton: Chapman and Hall/CRC. ISBN 978-0-412-31760-6.

McDermott, J., Schukken, Y., 1994. A review of methods used to adjust for cluster effects in explanatory epidemiological studies of animal populations. Preventive Veterinary Medicine 18, 155-173.

Jeong \& Revie, 2020 (Sampling salmon to estimate sea lice prevalence) 
Murray, A.G., 2002. Using observed load distributions with a simple model to analyse the epidemiology of sea lice (Lepeophtheirus salmonis) on sea trout (Salmo trutta). Pest Management Science 58, 585-594.

Nilsson, J., Folkedal, O., 2018. Sampling of Atlantic salmon Salmo salar from tanks and sea cages is size-biased. Aquaculture.

Norwegian Ministry of Industry and Fisheries, 2017. Forskrift om endring i forskrift om bekjempelse av lakselus. https://www.regjeringen.no/no/aktuelt/nye-regler-for-lusegrenser-omvaren/id2542196/. Norwegian Ministry of Industry and Fisheries, Norway.

Overton, K., Dempster, T., Oppedal, F., Kristiansen, T. S., Gismervik, K., Stien, L. H., 2018. Salmon lice treatments and salmon mortality in Norwegian aquaculture: a review. Reviews in Aquaculture. 11, 1398-1417.

Patanasatienkul, T., Sanchez, J., Rees, E.E., Krkošek, M., Jones, S.R., Revie, C.W., 2013. Sea lice infestations on juvenile chum and pink salmon in the Broughton Archipelago, Canada, from 2003 to 2012. Diseases of aquatic organisms, 105(2), pp.149-161.

Penston, M.J., Millar, C.P., Zuur, A., Davies, I.M., 2008. Spatial and temporal distribution of Lepeophtheirus salmonis (Kroyer) larvae in a sea loch containing Atlantic salmon, Salmo salar L., farms on the north-west coast of Scotland. Journal of Fish Diseases 31, 361-371.

Poulin, R., 2007. Are there general laws in parasite ecology? Parasitology 134, 763-776.

R Core Team 2019. R: A language and environment for statistical computing. R Foundation for Statistical Computing, Vienna, Austria. URL https://www.R-project.org/.

Revie, C., Dill, L., Finstad, B., Todd, C., 2009. Sea lice working group report. NINA special report 39, 1-117.

Revie, C.W., Gettinby, G., Treasurer, J., Wallace, C., 2005. Evaluating the effect of clustering when monitoring the abundance of sea lice populations on farmed Atlantic salmon. Journal of Fish Biology 66, 773-783.

Revie, C.W., Hollinger, E., Gettinby, G., Lees, F., Heuch, P., 2007. Clustering of parasites within cages on Scottish and Norwegian salmon farms: alternative sampling strategies illustrated using simulation. Preventive Veterinary Medicine 81, 135-147.

Samsing, F., Oppedal, F., Johansson, D., Bui, S., Dempster, T., 2014. High host densities dilute sea lice Lepeophtheirus salmonis loads on individual Atlantic salmon, but do not reduce lice infection success. Aquaculture Environment Interactions 6, 81-89.

SERNAPESCA, 2015. Resolution No. 013. Official Program for Surveillance and Control of Caligidosis (PSEVC-CALIGIDOSIS). Government of Chile, Development and Tourism, Undersecretary of Fisheries, National Service for Fisheries and Aquaculture.

Shaw, D., Dobson, A., 1995. Patterns of macroparasite abundance and aggregation in wildlife populations: a quantitative review. Parasitology 111, S111-S133.

Jeong \& Revie, 2020 (Sampling salmon to estimate sea lice prevalence) 
Torrissen, O., Jones, S., Asche, F., Guttormsen, A., Skilbrei, O.T., Nilsen, F., Horsberg, T.E., Jackson, D., 2013. Salmon lice-impact on wild salmonids and salmon aquaculture. Journal of Fish Diseases 36, 171-194.

Treasurer, J.W., Pope, James A, 2000. Selection of host sample number and design of a monitoring programme for ectoparasitic sea lice (Copepoda: Caligidae) on farmed Atlantic salmon, Salmo salar. Aquaculture 187, 247-260.

Vollset, K.W., Dohoo, I., Karlsen, Ø., Halttunen, E., Kvamme, B.O., Finstad, B., Wennevik, V., Diserud, O.H., Bateman, A., Friedland, K.D., Mahlum, S., Jørgensen, C., Qviller, L., Krkošek, M., Åtland, A., Barlaup, B.T., 2017. Disentangling the role of sea lice on the marine survival of Atlantic salmon. ICES Journal of Marine Science 75, 50-60.

Waddy, S., Burridge, L., Hamilton, M., Mercer, S., Aiken, D., Haya, K., 2002. Rapid communication/communication rapide emamectin benzoate induces molting in American lobster, Homarus americanus. Canadian Journal of Fisheries and Aquatic Sciences 59, 10961099. 


\section{List of figures}

Fig. 1. Relationship between the $\triangle \mathrm{AIC}=\left(\mathrm{AIC}_{\mathrm{nb}}-\mathrm{AIC} \mathrm{C}_{\mathrm{P}}\right)$ and the dispersion parameter $(\kappa)$ of the negative binomial distribution. These values were estimated from sea lice counts taken on salmon farms in four areas; two in Norway, one in New Brunswick (NB), Canada, and one in Chile. (The y-axis is on a log scale.)

Fig. 2. Relationships between abundance and prevalence of sea lice on farmed salmon. The relationships were calculated with Equation 1. (A) The theoretical relationship that is hypothesised to hold between abundance and prevalence, assuming a negative binomial distribution for a range of values that might hold for the dispersion parameter $\kappa$. (B) The empirical relationship between sea lice abundance and prevalence based on data from salmon farms in four areas. The black line and narrow dark grey area indicate an estimated mean of 2.19 and a $95 \%$ confidence interval [1.76 - 2.89] for $\kappa$, with the wider light grey area representing the prediction band [0.8 8] for $\kappa$.

Fig. 3. Contour plots from Monte-Carlo simulations illustrating the impact of various true prevalence and intraclass correlation (ICC) values on estimated prevalence under different sampling regimes. Various combinations of fish sample size per pen and number of pens were simulated to obtain estimated accuracies, taken as the average deviation of prevalence estimated from sampled fish from the prevalence estimated from all fish. Deviations were calculated through $\frac{\mid \text { estimated prevalence-true prevalence| }}{\text { true prevalence }}$. The dashed lines indicate scenarios of 'equivalent sampling effort' (i.e. the combination of fish sample size per pen and number of pens that will result in a total of 40 fish being sampled).

Fig. 4. Plots from Monte-Carlo simulations illustrating the impact of various true prevalence and intraclass correlation (ICC) values on the detection of treatment efficacies through sampling. Various combinations of fish sample size per pen and number of pens were simulated to estimate the minimum requirements to detect three levels of treatment efficacy. Treatment efficacy was assumed to be $1-\frac{\text { post-treatment prevalence }}{\text { pre-treatment prevalence }}$. The combinations represent the required sampling for the detection of difference with $80 \%$ power and 95\% confidence level. "True Prev. (Pre-TX)" indicates true prevalence before a treatment is applied. The black dashed lines indicate scenarios of equivalent sampling effort. 


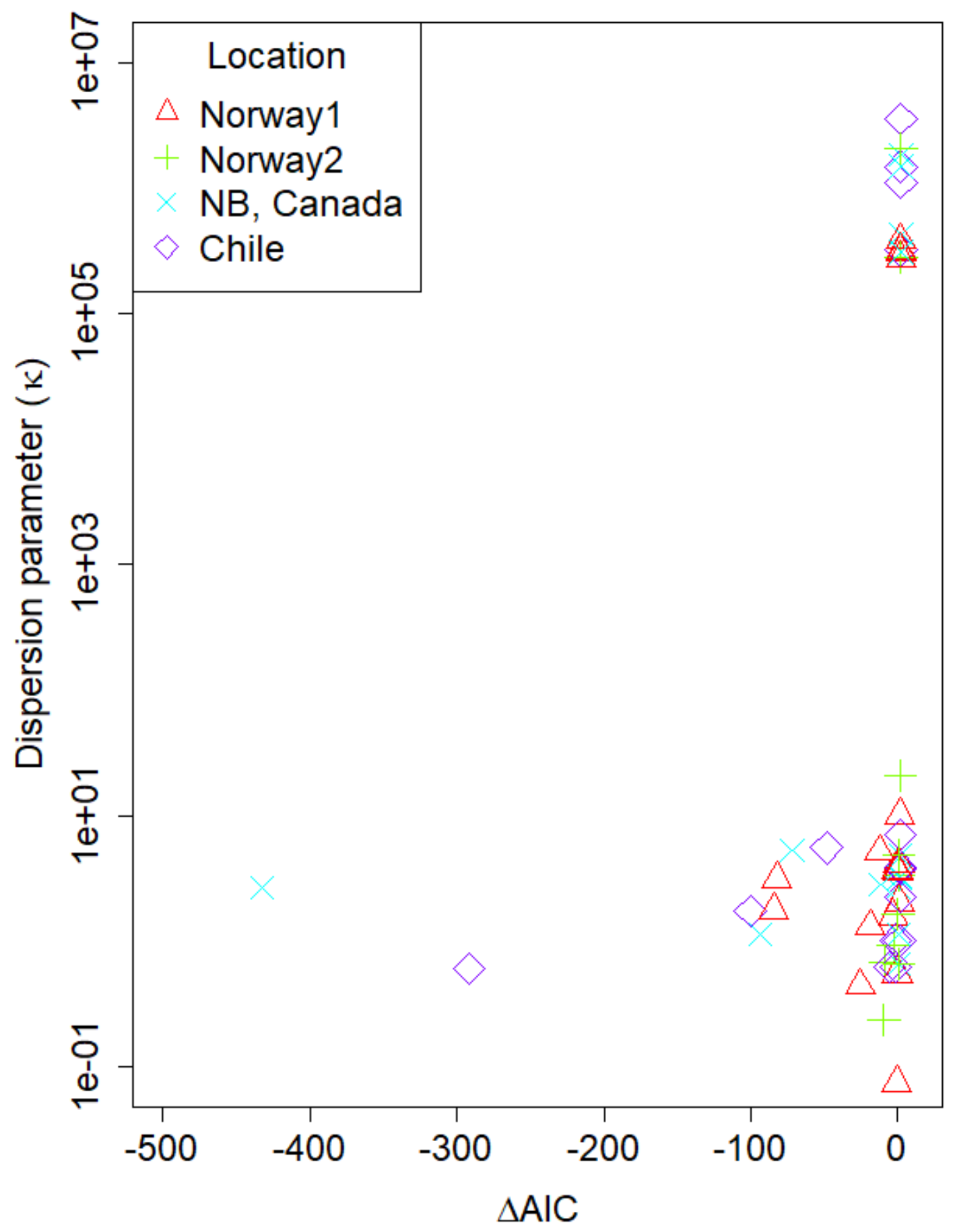

Fig. 1

Jeong \& Revie, 2020 (Sampling salmon to estimate sea lice prevalence) 
(A)

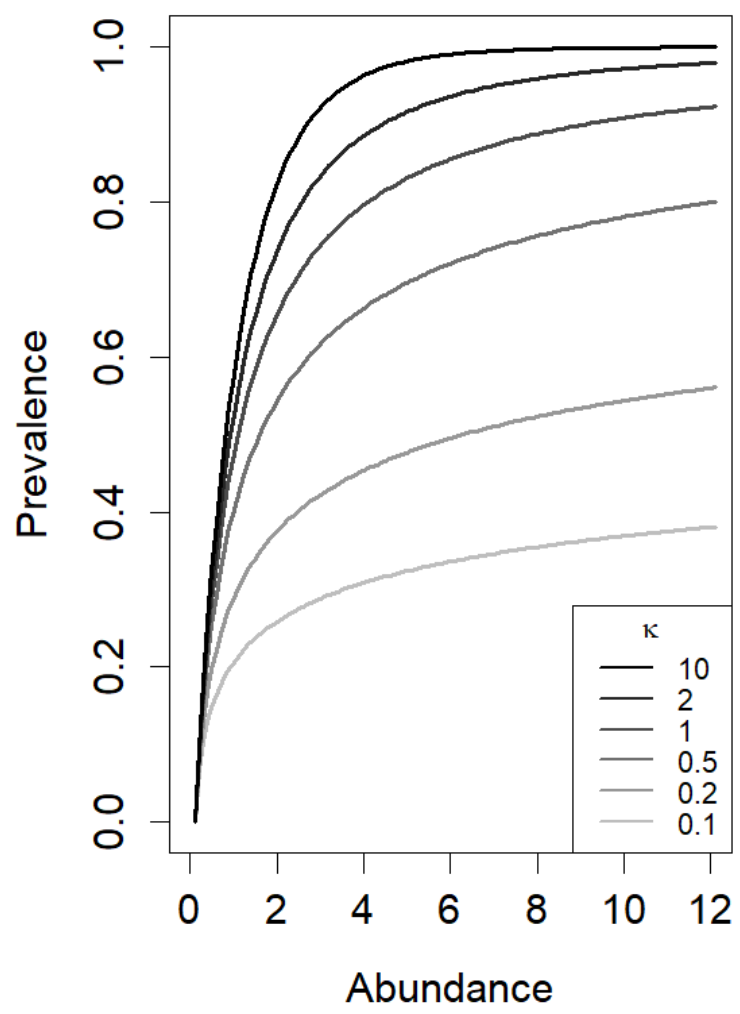

(B)

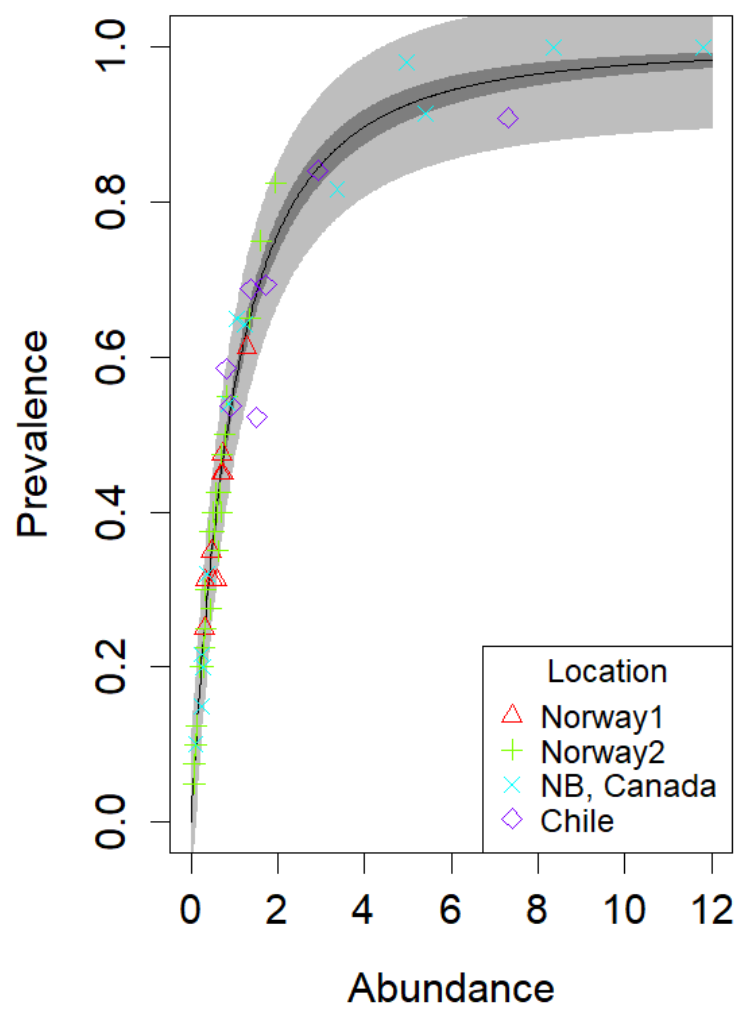

Fig. 2 

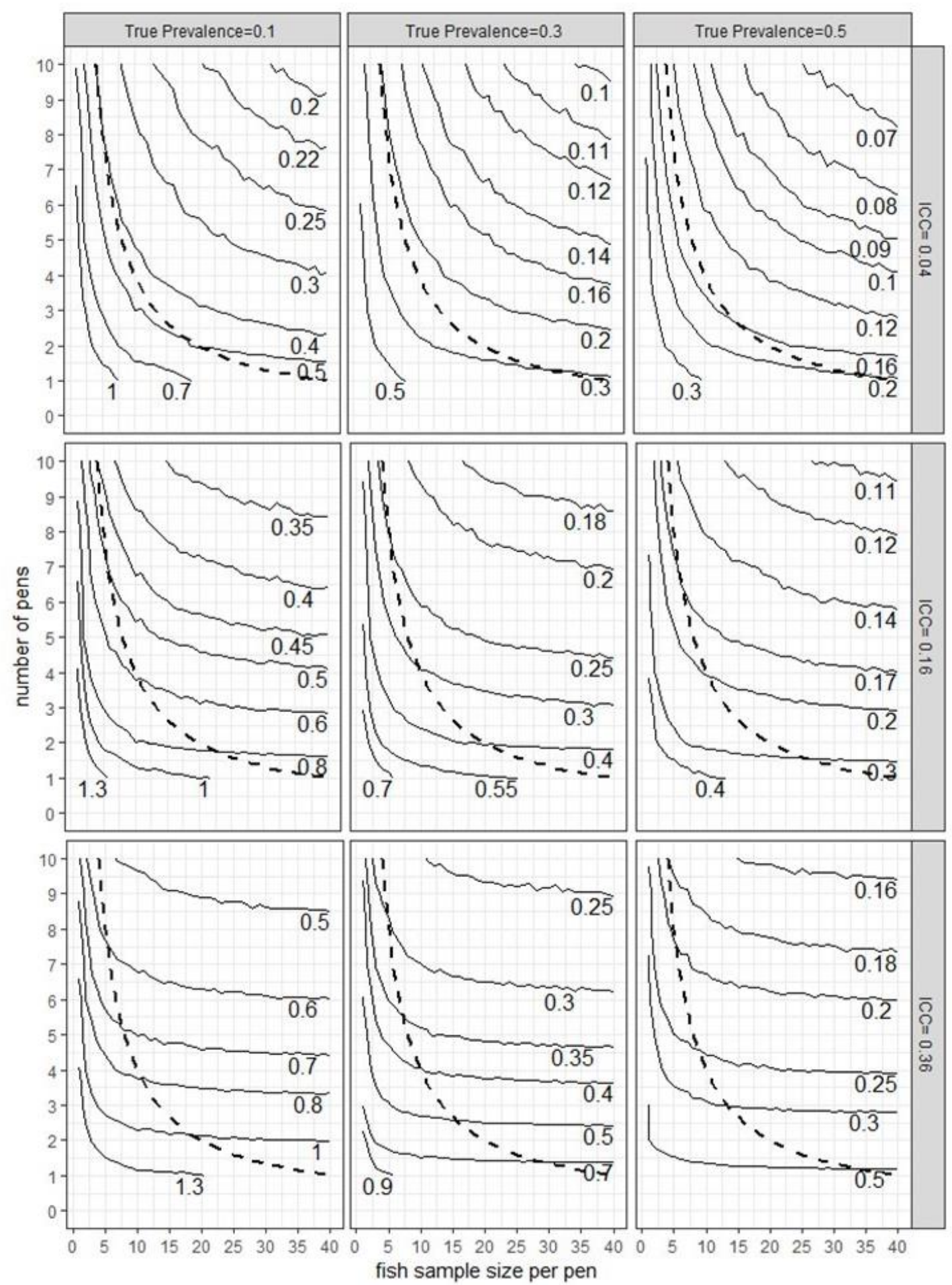

Fig. 3

Jeong \& Revie, 2020 (Sampling salmon to estimate sea lice prevalence) 

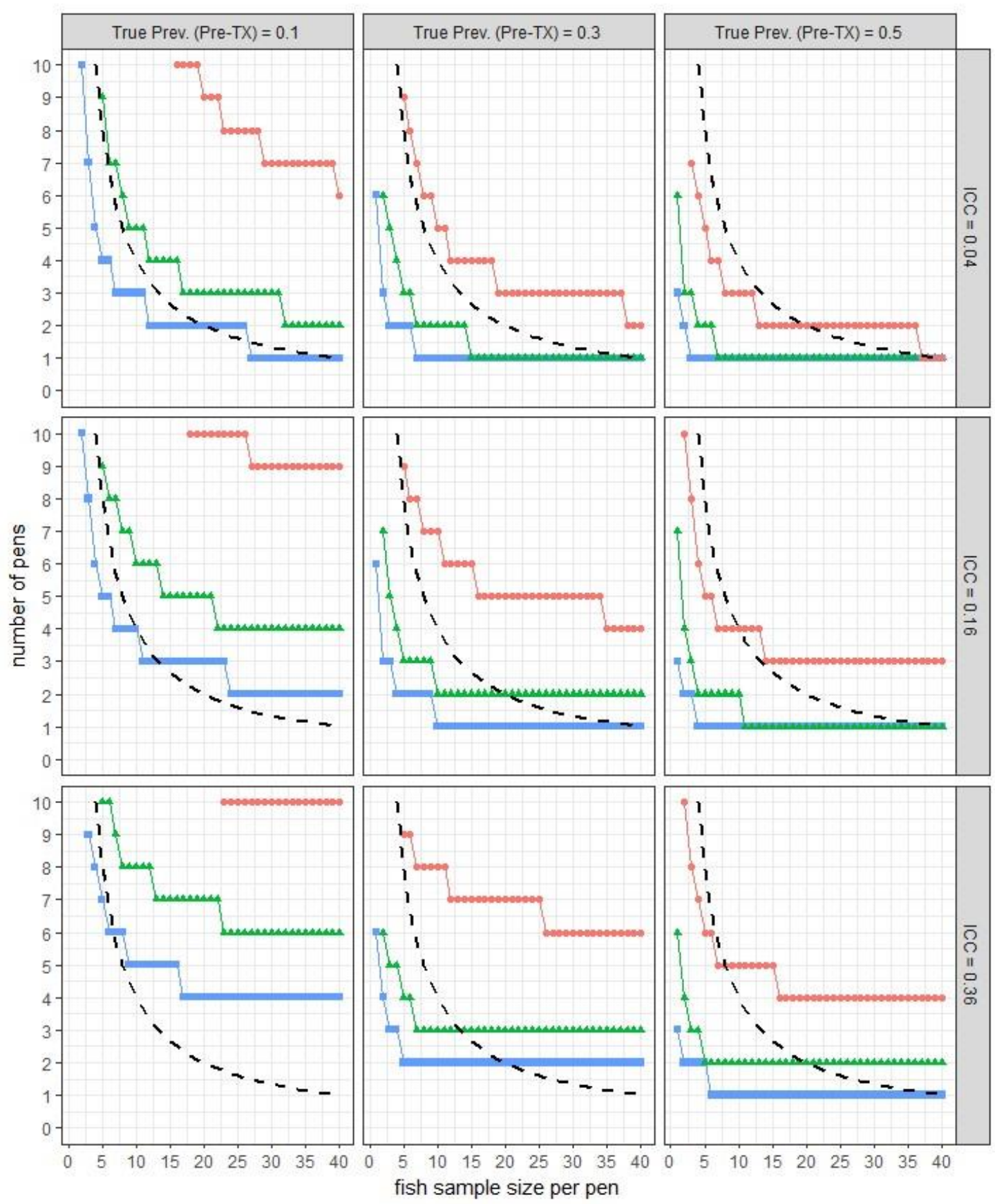

Treatment.efficacy $\rightarrow 0.3 \rightarrow 0.6 \rightarrow 0.9$

Fig. 4 